\title{
Delzant's $T$-invariant, Kolmogorov complexity and one-relator groups
}

Ilya Kapovich and Paul Schupp*

\begin{abstract}
We prove that for "random" one-relator groups the Delzant $T$-invariant (which measures the smallest size of a finite presentation of a group) is comparable in magnitude with the length of the defining relator. The proof relies on our previous results regarding isomorphism rigidity of generic one-relator groups and on the methods of the theory of Kolmogorov-Chaitin complexity.
\end{abstract}

Mathematics Subject Classification (2000). Primary 20F36; Secondary 20E36, 68Q30, 03D.

Keywords. Delzant's $T$-invariant, Kolmogorov complexity, generic groups.

\section{Introduction}

Delzant [14] introduced an extremely interesting but still rather enigmatic invariant for finitely presentable groups. For any finite presentation $\Pi=\langle X \mid R\rangle$ define the length $\ell(\Pi)$ as

$$
\ell(\Pi):=\sum_{r \in R} \max \{|r|-2,0\} .
$$

If $G$ is a finitely presentable group, the T-invariant $T(G)$ of $G$, which we also call the presentation rank of $G$, is defined [14] as

$$
T(G):=\min \{\ell(\Pi) \mid \Pi \text { is a finite presentation of the group } G\} .
$$

The $T$-invariant plays a central role in Delzant and Potyagailo's proof of the strong accessibility (or "hierarchical decomposition") theorem for finitely presented groups [16]. This theorem is the strongest and most difficult of numerous accessibility results ([17], [18], [5], [6], [40], [15], [42], [31]). One can also define a closely related notion, the non-reduced $T$-invariant $T_{1}(G)$, as the minimum of sums of lengths of the

\footnotetext{
*The authors were supported by the NSF grant DMS\#0404991 and the NSA grant DMA\#H98230-04-1-0115.
} 
defining relators, taken over all finite presentations of $G$. As we observe in Lemma 5.2 below, if $G$ is a finitely presentable group without elements of order two then

$$
T(G) \leq T_{1}(G) \leq 3 T(G) .
$$

The non-reduced $T$-invariant has been studied in the context of 3-manifolds, where it turns out to be related to the notion of Matveev complexity. We refer the reader to a recent paper of Pervova and Petronio [37] for a discussion on this subject.

If $G$ is a finitely generated group then the ordinary $\operatorname{rank}, \operatorname{rk}(G)$, of $G$ is the smallest cardinality of a finite generating set for $G$. The first (and already quite nontrivial) accessibility result is Grushko's theorem [26] which asserts that for finitely generated groups $G_{1}$ and $G_{2}$ we have $\operatorname{rk}\left(G_{1} * G_{2}\right)=\operatorname{rk}\left(G_{1}\right)+\operatorname{rk}\left(G_{2}\right)$. In [14] Delzant proved a similar theorem for the presentation rank, namely that

$$
T\left(G_{1} * G_{2}\right)=T\left(G_{1}\right)+T\left(G_{2}\right)
$$

if $G_{1}, G_{2}$ are finitely presentable groups.

The hierarchical decomposition theorem proved in [16] implies, for example, that an iterated process of JSJ-decomposition (in any sense of the word), see [41], [38], [19], [21], [7], applied to a finitely presented group, then to the factors of its JSJ-decomposition, and so on, always terminates. The $T$-invariant is also crucial in Delzant's generalization [15] of Sela's acylindrical accessibility result [40] for finitely presented groups.

If $\Pi$ is a finite presentation, let $G(\Pi)$ be the group defined by $\Pi$. We can regard $T$ as a function defined over finite presentations by setting $T(\Pi)=T(G(\Pi))$. If $G$ is given by a particular finite presentation $\Pi$ then $\ell(\Pi)$ gives an obvious upper bound for $T(G(\Pi))$. However, it is very unclear in general how to estimate $T(G)$ from below. For example, if $\Pi=\langle X \mid R\rangle$ and $\alpha \in \operatorname{Aut}(F(X))$ then the presentations $\Pi$ and $\Pi^{\prime}=\langle X \mid \alpha(R)\rangle$ define isomorphic groups but it is easy to produce examples where $\ell\left(\Pi^{\prime}\right)$ is arbitrarily smaller than $\ell(\Pi)$.

We prove however that for "most" one-relator presentations this does not happen and that the value of Delzant's $T$-invariant is comparable in magnitude with the length of the defining relator. If $r \in F\left(a_{1}, \ldots, a_{k}\right)$, let $G_{r}:=\left\langle a_{1}, \ldots, a_{k} \mid r\right\rangle$ be the one-relator group whose defining relator is $r$. Our main result is:

Theorem A. Fix an integer $k>1$ and let $F=F\left(a_{1}, \ldots, a_{k}\right)$. For any number $0<\varepsilon<1$ there is an integer $n_{1}>0$ and a constant $M=M(k, \varepsilon)>0$ with the following property.

Let $J$ be the set of all nontrivial cyclically reduced words $r$ such that

$$
T\left(G_{r}\right) \log _{2} T\left(G_{r}\right) \geq M|r| .
$$

Then for any $n \geq n_{1}$

$$
\frac{\#\{r \in J:|r|=n\}}{\#\{r \in F: r \text { is cyclically reduced and }|r|=n\}} \geq 1-\varepsilon .
$$


Thus for any fixed $0<\varepsilon, \delta<1$ we asymptotically have $T\left(G_{r}\right) \geq c|r|^{1-\delta}$, where $c$ is a constant, for at least the fraction $(1-\varepsilon)$ of all cyclically reduced words $r$ of a given length. This says that the description of a one-relator group by a generic relator $r$ is "essentially incompressible". In view of the above remarks about the connection between $T(G)$ and $T_{1}(G)$, the same conclusion as in Theorem A also holds for $T_{1}\left(G_{r}\right)$.

This is a good place to observe that the function $T$ is not computable.

Observation 1.1. The function $T$, as a function over finite presentations, is not a computable function.

Proof. We say that a finitely generated group $G$ is essentially free if $G$ is the free product of a finitely generated free group and finitely many cyclic groups of order two. The only defining relators in the "standard presentation" $\Pi_{0}$ of such a group are the squares of those generators which have order two and so $\ell\left(\Pi_{0}\right)=0$.

It is easy to use Tietze transformations to show that any group $G$ having a finite presentation in which all relators have length at most two is essentially free. Hence, by the definition of $T(G)$, a finitely presentable group $G$ has $T(G)=0$ if and only if $G$ is essentially free.

Recall that a property $\mathcal{P}$ of finitely presented groups is a Markov property if $\mathcal{P}$ is independent of presentation, there are finitely presented groups with $\mathcal{P}$ and there is a finitely presented group $G_{*}$ such that $G_{*}$ cannot be embedded in any finitely presented group with $\mathcal{P}$. Being essentially free is clearly a Markov property. We can take $G_{*}$ to be the cyclic group of order three. The classic Adian-Rabin Theorem [32] says that if $\mathcal{P}$ is any Markov property then there is no algorithm over all finite presentations which, when given a finite presentation $\Pi$, decides whether or not the group $G(\Pi)$ has $\mathcal{P}$.

If the function $T$ were computable then, for any finite presentation $\Pi$, we could decide the essential freeness of $G(\Pi)$ by computing $T(\Pi)$. Hence $T$ cannot be computable.

The proof of Theorem A involves several different probabilistic tools. The idea introduced in this paper is the use of Kolmogorov complexity, a concept that plays an important role in coding theory, algorithmic probability and complexity theory. This notion is also sometimes known as "Kolmogorov-Chaitin complexity" because of the contributions of Chaitin to the subject. Roughly speaking, the Kolmogorov complexity of a word is the size of the smallest computer program (in a fixed programming language) that can compute the given word. Surprisingly, the only previous use of Kolmogorov complexity in group theory known to us is a 1985 paper of Grigorchuk [22], giving an interesting application of Kolmogorov complexity to algorithmic problems in group theory. 
Our results here also depend on [29] and [30] where we obtained a number of results regarding a very strong Mostow-type "isomorphism rigidity" for generic onerelator groups. These results use a combination of the Arzhantseva-Ol'shanskii minimization technique and their ingenious "non-readability" small cancellation condition [29] and Large Deviation Theory [30] to study the behavior of random words under an arbitrary automorphism of the ambient free group. The isomorphism rigidity theorems proved in [30] allow us, given any finite presentation $\Pi=\langle X \mid R\rangle$ defining a group isomorphic to $G_{r}=\left\langle a_{1}, \ldots, a_{k} \mid r\right\rangle$ (where $k>1$ is fixed) for a generic relator $r$ plus a small initial segment $u$ of $r$, to algorithmically recover the word $r$. This implies that $r$ is uniquely algorithmically determined by an amount $O(\ell(\Pi) \log \ell(\Pi))$ of information. (The logarithmic term comes from the fact that the subscripts in the enumeration of letters in $X$ also need to be encoded.) From here one can deduce that the Kolmogorov complexity of $r$ is $\leq O(\ell(\Pi) \log \ell(\Pi))$. On the other hand, using the methods of algorithmic probability, in particular the notion of prefix complexity, we can deduce that a cyclically reduced word $r$ of a given length has Kolmogorov complexity $\geq c|r|$ asymptotically with probability $\geq 1-\varepsilon$. These inequalities taken together yield the conclusion of Theorem A.

We believe that the general analogue of Theorem A is true. This would say that if we fix a number $k \geq 2$ of generators and any number $m \geq 1$ of defining relators, then a generic $k$-generator $m$-relator presentation should essentially be the shortest description of the group defined. We have seen that the proof of Theorem A relies on two components: the Kolmogorov complexity arguments used in this paper and the isomorphism rigidity results for random one-relator groups established in [29], [30]. Now most of the arguments and statements of [29], [30] needed to prove isomorphism rigidity actually go through for generic groups with an arbitrary fixed number of relators and we believe that "generic groups are rigid" in general. However, to actually infer rigidity, at the end of the proof we use a crucial fact about one-relator groups. Namely, we need the classical theorem of Magnus (see, for example, [32]) which says that if two elements $r$ and $s$ have the same normal closures in a free group $F$ then $r$ is conjugate in $F$ to $s$ or $s^{-1}$. This statement does not hold in general for tuples consisting of more than one element of $F$. However, we believe that the desired analogue does hold generically.

If $\tau=\left(u_{1}, \ldots, u_{m}\right)$ is an $m$-tuple of elements of the free group $F_{k}$, the symmetrized set $R(\tau)$ generated by $\tau$ consists of all the cyclic permutations of cyclically reduced forms of $u_{i}^{ \pm 1}$.

Conjecture 1.2 (Stability Conjecture). Fix $k \geq 2$ and $m \geq 1$. Let $F=F\left(a_{1}, \ldots, a_{k}\right)$. Then there exists an algorithmically recognizable generic class $\mathcal{C}$ of $m$-tuples of elements of $F$ with the following property. If $\sigma, \tau \in \mathcal{C}$ and $\alpha \in \operatorname{Aut}(F)$ are such that $R(\sigma)$ and $R(\alpha(\tau))$ have the same normal closure in $F$ then $R(\sigma)=R(\alpha(\tau))$. 
Magnus' theorem implies that the Stability Conjecture holds for $m=1$ with $\mathcal{C}=F_{k}$. If one could establish the Stability Conjecture, then both the isomorphism rigidity results of [30] and the results of this paper would then follow for finitely presented groups with any fixed numbers of generators and relators exactly as in the one-relator case.

In [30] we showed that for a fixed $k \geq 2$ the number $I_{n}$ of isomorphism types of $k$-generator one-relator groups with cyclically reduced defining relators of length $n$ satisfies

$$
\frac{c_{1}(2 k-1)^{n}}{n} \leq I_{n} \leq \frac{c_{2}(2 k-1)^{n}}{n},
$$

where $c_{1}=c_{1}(k)>0, c_{2}=c_{2}(k)>0$ are some constants independent of $n$. Using auxiliary results from the proof of Theorem A we obtain an improvement of this estimate in the present paper and compute the precise asymptotics of $I_{n}$ :

Theorem B. Let $k \geq 2$ be a fixed integer. Then the number $I_{n}$ of isomorphism types of $k$-generator one-relator groups with cyclically reduced defining relators of length $n$ satisfies:

$$
I_{n} \sim \frac{(2 k-1)^{n}}{n k ! 2^{k+1}} .
$$

Here $f(n) \sim g(n)$ means that $\lim _{n \rightarrow \infty} f(n) / g(n)=1$.

The authors are grateful to Carl Jockusch and Paul Vitanyi for helpful discussions regarding Kolmogorov complexity. They also thank Warren Dicks for suggesting the problem of computing the precise asymptotics of $I_{n}$.

\section{Kolmogorov complexity}

The $T$-invariant is a measure of "smallest descriptive complexity" in the framework of finite presentations of groups while Kolmogorov complexity is a general theory of "minimal descriptive complexity". We provide here only a brief discussion of the relevant facts regarding Kolmogorov complexity and refer the reader to the survey of Fortnow [20] for an overview and to the excellent and comprehensive book of $\mathrm{Li}$ and Vitanyi [34] for detailed background information.

Intuitively speaking, the Kolmogorov complexity $C(x)$ of a finite binary string $x$ is the size of the smallest computer program $M$ that can compute $x$. In order for this notion to make sense one needs to first fix a "programming language" but it turns out that all reasonable choices yield measures which are equivalent up to an additive constant.

Note that $C(x)$, as a measure of descriptive complexity of $x$, totally disregards how long the particular program $M$ will have to run in order to compute $x$. Some 
strings clearly admit much shorter descriptions then their length. For example, if $x_{0}$ is the binary representation of the number $2^{2^{2^{10}}}$ then the length of $x_{0}$ is huge, namely $1+2^{2^{10}}$. Yet we were just able to give a very short unambiguous description of $x_{0}$. Thus $x_{0}$ has small Kolmogorov complexity and $C\left(x_{0}\right) \ll\left|x_{0}\right|$. On the other hand it is intuitively clear that for a "random" string $x$ of large length, the shortest description of $x$ is essentially $x$ itself. In this case $C(x) \approx|x|$. This phenomenon is called "incompressibility" and plays an important role in complexity theory for establishing lower complexity bounds of various algorithms.

Recall that any Turing machine $M$ on the set of finite binary strings $\{0,1\}^{*} \mathrm{com}$ putes a partial recursive function $\{0,1\}^{*} \rightarrow\{0,1\}^{*}$ and, moreover, every partial recursive function $\{0,1\}^{*} \rightarrow\{0,1\}^{*}$ arises in this fashion.

Once one has fixed the formalism of Turing machines, one can identify a Turing machine with its sequence of instructions and think of Turing machines as programs. A Turing machine $M$ can then itself be coded as a binary string according to some fixed effective method and we write $\langle M\rangle$ for the code of the machine $M$. The pair consisting of a Turing machine $M$ and an input $w$ can then be given the code $\langle M\rangle w$. A basic feature of the theory of computability is the existence of a universal Turing machine $U$, which, if its input is a code $\langle M\rangle w$, simulates $M$ on input $w$. To be more precise, a Turing machine $U$ is universal if for any Turing machine $M$ there is a binary string $\langle M\rangle$ such that for any string $w \in\{0,1\}^{*}$ the machine $U$ produces the same result on input $\langle M\rangle w$ as $M$ does on $w$.

Definition 2.1. Fix a universal Turing machine $U$ with the alphabet $\Sigma:=\{0,1\}$. Then $U$ computes a universal partial recursive function $\phi$ from $\Sigma^{*}$ to $\Sigma^{*}$. That is, for any partial recursive function $\psi$ there is a string $z \in \Sigma^{*}$ such that for all $x \in \Sigma^{*}$, $\phi(z x)=\psi(x)$.

For a finite binary string $x \in \Sigma^{*}$ we define the Kolmogorov complexity $C(x)$ as

$$
C(x):=\min \left\{|p|: p \in \Sigma^{*}, \phi(p)=x\right\} .
$$

Kolmogorov complexity is traditionally defined for finite binary strings. However, if $s>1$ is a fixed integer, then all of the standard definitions and theorems go through essentially unchanged if one considers finite strings $x$ in a fixed $s$-letter alphabet $A$. This can be done in either of two essentially equivalent ways. First, one can modify Definition 2.1 by choosing $U$ to be a universal Turing machine with the alphabet $A_{s}$ computing a universal partial recursive function from $A_{s}^{*}$ to $A_{s}^{*}$. Alternatively, one can fix a recursive bijection $h: A_{s}^{*} \rightarrow \Sigma^{*}$ and define $C_{s}(x)$, where $x \in A_{s}^{*}$ to be $C(h(x))$. We choose the latter option since most theorems in [34] are stated for binary strings and we want to be able to cite the results of [34] verbatim.

Definition 2.2. Let $s>1$ be an integer and let $A_{s}$ be an alphabet with $s$ letters. Fix a recursive bijection $h: A_{s}^{*} \rightarrow\{0,1\}^{*}$. 
For any string $x \in A_{s}^{*}$ define its Kolmogorov complexity $C_{s}(x)$ as

$$
C_{s}(x):=C(h(x)) \text {. }
$$

Kolmogorov complexity lacks some mathematical properties which are essential for certain arguments. Fortunately, this difficulty can be overcome by using the closely related notion of prefix complexity. For a detailed discussion of this notion we refer the reader to Chapters 2 and 3 of [34]. In the present paper we need only cite a few basic facts regarding prefix complexity from [34]. A partial recursive function $\phi$ on $\Sigma^{*}$ is called a prefix function if whenever $\phi(x)$ is defined and $x$ is a proper initial segment of $y$, then $\phi(y)$ is undefined. There is a corresponding notion of a prefix machine. Informally speaking, a prefix machine does not require an "end-of-tape" symbol for the input word and decides whether or not to halt only based on its current state and before scanning the next letter of the input. The machine starts working on an infinite input word and, after performing a computational step on the working and output tapes, the machine either moves one letter to the right on the input tape or halts and terminates its work.

Just as with ordinary Turing machines, there exist universal prefix machines computing universal prefix partial recursive functions (see Theorem 3.1.1 in [34]).

Definition 2.3. Fix a universal prefix Turing machine $U^{\prime}$ with the alphabet $\Sigma=\{0,1\}$. Then $U^{\prime}$ computes a universal prefix partial recursive function $\psi$ from $\Sigma^{*}$ to $\Sigma^{*}$.

For a finite binary string $x \in \Sigma^{*}$ we define the prefix complexity $K(x)$ as

$$
K(x):=\min \left\{|p|: p \in \Sigma^{*}, \psi(p)=x\right\}
$$

Similarly to the case of Kolmogorov complexity, prefix complexity can be defined not only for binary but also for $s$-ary strings.

Definition 2.4. Let $s>1$ be an integer and let $A_{s}$ be an alphabet with $s$ letters. Fix the same recursive bijection $h: A_{s}^{*} \rightarrow\{0,1\}^{*}$ as in Definition 2.2.

For any string $x \in A_{s}^{*}$ define its prefix complexity $K_{s}(x)$ as

$$
K_{S}(x):=K(h(x)) .
$$

For our purposes, the crucial way in which prefix complexity is better than Kolmogorov complexity is that $\sum_{x \in\{0,1\}^{*}} 2^{-K(x)} \leq 1$ while $\sum_{x \in\{0,1\}^{*}} 2^{-C(x)}$ diverges.

We list here some relevant properties of Kolmogorov and prefix complexity.

Proposition 2.5. Let $s>1$ be a fixed integer and let $A_{s}$ be an $s$-letter alphabet. Then the following holds. 
(1) We have

$$
\sum_{x \in\{0,1\}^{*}} 2^{-K(x)} \leq 1
$$

(2) Up to additive constants, for any $x \in\{0,1\}^{*}$ we have

$$
C(x) \leq K(x) \leq C(x)+\log _{2} C(x) .
$$

(3) We have

$$
\sum_{x \in A_{s}^{*}} 2^{-K_{s}(x)} \leq 1
$$

(4) Up to additive constants for any $x \in A_{s}^{*}$ we have

$$
C_{s}(x) \leq K_{S}(x) \leq C_{s}(x)+\log _{2} C_{s}(x) .
$$

Proof. Part (1), as observed by Levin [33], is a direct corollary of Kraft's Inequality, which is ubiquitous in information theory (see also 4.2.2(b) in [34]). Part (2) is statement 3.1.3 in [34]. Clearly, (1) implies (3) and, also, (2) implies (4). Since part (1) is quite important for our purposes, we provide a proof here.

A subset $S \subseteq\{0,1\}^{*}$ is prefix-free if whenever $p, q \in S, p \neq q$ then $p$ is not an initial segment of $q$. Recall that by definition $K(x)$ is the shortest length of a prefix program $p$ with $\psi(p)=x$. Thus the set $S$ of such all such $p$ corresponding to $x \in\{0,1\}^{*}$ is prefix-free. If $p$ is a binary string, then $2^{-|p|}$ is the Lebesgue measure of the subset $S_{p}$ of the unit interval $I=[0,1]$ consisting of those numbers whose binary expansion begins with $p$. Since $S$ is prefix-free, subsets $S_{p}$ and $S_{q}$ are disjoint for $p \neq q$. The inequality thus follows from the countable additivity of Lebesgue measure.

We also recall the classical Markov inequality from probability theory which can be found in most probability textbooks (see, for example, Lemma 1.7.1 in [39]):

Lemma 2.6 (Markov Inequality). Let $X: \Omega \rightarrow \mathbb{R}$ be a nonnegative random variable on a sample probability space $\Omega$ with the expected value $E(X)>0$. Then for any $\delta>0$ we have

$$
P(X \geq \delta) \leq \frac{E(X)}{\delta} .
$$

Lemma 2.7. Let $s>1$ be a fixed integer and let $A_{s}$ be an $s$-letter alphabet. Let $\Omega \subset A_{s}^{*}$ be a nonempty subset equipped with a discrete non-vanishing probability measure $\Pi$, so that $\sum_{x \in \Omega} P(\{x\})=1$. Denote $\mu(x):=P(\{x\})$ for any $x \in \Omega$.

Then for any $\delta>0$ we have

$$
P\left(K_{s}(x) \geq-\log _{2} \mu(x)-\log _{2} \delta\right)=P\left(2^{-K_{s}(x)} \leq \delta \mu(x)\right) \geq 1-\frac{1}{\delta} .
$$


Proof. Consider the function $X: \Omega \rightarrow \mathbb{R}$ defined by $X(x)=\frac{2^{-K_{S}(x)}}{\mu(x)}$.

The $\Pi$-expected value of $X$ is

$$
E(X)=\sum_{x \in \Omega} \mu(x) \frac{2^{-K_{s}(x)}}{\mu(x)} \leq \sum_{x \in \Omega} 2^{-K_{s}(x)} \leq \sum_{x \in A_{s}^{*}} 2^{-K_{s}(x)} \leq 1,
$$

where the last inequality holds by Proposition 2.5.

Therefore by Markov's inequality

$$
P\left(\frac{2^{-K_{s}(x)}}{\mu(x)} \geq \delta\right) \leq \frac{E(X)}{\delta} \leq \frac{1}{\delta}
$$

and so

$$
P\left(\frac{2^{-K_{s}(x)}}{\mu(x)} \leq \delta\right) \geq P\left(\frac{2^{-K_{s}(x)}}{\mu(x)}<\delta\right) \geq 1-\frac{1}{\delta}
$$

as required.

\section{Kolmogorov complexity and freely reduced words}

Convention 3.1. Let $k>1$ and let $F=F\left(a_{1}, \ldots, a_{k}\right)$. Put

$$
A_{2 k}:=\left\{a_{1}, \ldots, a_{k}, a_{1}^{-1}, \ldots, a_{k}^{-1}\right\} .
$$

As usual we identify $F$ with the set of all freely reduced words in $A_{2 k}^{*}$. Thus if $g \in F$ then $|g|$ is the length of the unique freely reduced word representing $g$. For a subset $S \subseteq F$ denote by $\gamma(n, S)$ the number of all $x \in S$ with $|x|=n$. Similarly, denote by $\rho(n, S)$ the number of all $x \in S$ such that $|x| \leq n$. Note that $\gamma(n, F)=2 k(2 k-1)^{n-1}$ for $n \geq 1$. Denote by CR the set of all cyclically reduced words in $A_{2 k}^{*}$. Thus $\mathrm{CR} \subseteq F$. These notations will be fixed for the remainder of the paper, unless specified otherwise.

The next result is is easy to see.

Lemma 3.2 ([30]). For any $n \geq 1$ we have

$$
(2 k-1)^{n} \leq \gamma(n, \mathrm{CR}) \leq 2 k(2 k-1)^{n} .
$$

Moreover, in Proposition 5.8 below we will see an explicit formula for $\gamma(n, \mathrm{CR})$, which we do not need for the moment. 
Proposition 3.3. Let $c \geq 1$. Denote by $Z$ the set of all cyclically reduced words $x$ such that

$$
C_{2 k}(x) \geq-\frac{c}{2}+|x| \frac{\log _{2}(2 k-1)}{2} .
$$

Then there is $n_{0}>1$ such that for any $n \geq n_{0}$ we have

$$
\frac{\gamma(n, Z)}{\gamma(n, \mathrm{CR})} \geq 1-\frac{1}{2^{c}}
$$

Proof. Let $n>0$ be an integer and let $W_{n}$ be the set of all cyclically reduced words of length $n$ with the uniform discrete probability measure $P$. As in Lemma 2.7 denote $\mu(x):=P(\{x\})$ for any $x \in W_{n}$. Then by Lemma 3.2 for any $x \in \mathcal{W}_{n}$ we have

$$
\frac{1}{2 k}(2 k-1)^{-n} \leq P(\{x\})=\mu(x)=\frac{1}{\gamma(n, \mathrm{CR})} \leq(2 k-1)^{-n} .
$$

We apply Lemma 2.7 with $\delta=2^{c}$. Hence

$$
\begin{aligned}
1-\frac{1}{2^{c}} & \leq P\left(2^{-K_{2 k}(x)} \leq 2^{c} \mu(x)\right) \\
& \leq P\left(2^{-K_{2 k}(x)} \leq 2^{c}(2 k-1)^{-n}\right) \\
& =P\left(-K_{2 k}(x) \leq c-n \log _{2}(2 k-1)\right) \\
& =P\left(K_{2 k}(x) \geq-c+n \log _{2}(2 k-1)\right) .
\end{aligned}
$$

Recall that by Proposition 2.5

$$
K_{2 k}(x) \leq C_{2 k}(x)+\log _{2} C_{2 k}(x)+c_{0}
$$

where $c_{0}$ is some fixed constant. There is $n_{0}>1$ such that for any word $x \in A_{2 k}^{*}$ of length $n \geq n_{0}$ we have

$$
K_{2 k}(x) \leq 2 C_{2 k}(x)
$$

Therefore if $n \geq n_{0}$ then

$$
\begin{aligned}
1-\frac{1}{2^{c}} & \leq P\left(K_{2 k}(x) \geq-c+n \log _{2}(2 k-1)\right) \\
& \leq P\left(C_{2 k}(x)+\log _{2} C_{2 k}(x)+c_{0} \geq-c+n \log _{2}(2 k-1)\right) \\
& \leq P\left(2 C_{2 k}(x) \geq-c+n \log _{2}(2 k-1)\right),
\end{aligned}
$$

as required. 


\section{Genericity in free groups}

If $b_{n}, b \in \mathbb{R}$ and $\lim _{n \rightarrow \infty} b_{n}=b$, we say that the convergence is exponentially fast if there exist $C>0$ and $\sigma$ with $0<\sigma<1$ such that for all $n$ we have

$$
\left|b_{n}-b\right| \leq C \sigma^{n}
$$

Definition 4.1. Let $S \subseteq Q \subseteq F$. We say that $S$ is $Q$-generic if

$$
\lim _{n \rightarrow \infty} \frac{\rho(n, S)}{\rho(n, Q)}=1
$$

If in addition the convergence in the above limit is exponentially fast, we say that $S$ is exponentially $Q$-generic.

Similarly, $S$ is called (exponentially) $Q$-negligible if $Q-S$ is (exponentially) $Q$-generic.

Note that the union of two (exponentially) $Q$-negligible sets is (exponentially) $Q$ negligible and the intersection of two (exponentially) $Q$-generic sets is (exponentially) $Q$-generic.

Proposition 4.2 ([30]). The following hold:

(1) A subset $S \subseteq F$ is exponentially $F$-negligible if and only if

$$
\lim _{n \rightarrow \infty} \frac{\gamma(n, S)}{(2 k-1)^{n}}=0
$$

with exponentially fast convergence.

(2) A subset $S \subseteq \mathrm{CR}$ is exponentially $\mathrm{CR}$-negligible if and only if

$$
\lim _{n \rightarrow \infty} \frac{\gamma(n, S)}{(2 k-1)^{n}}=0
$$

with exponentially fast convergence.

(3) A subset $Q \subseteq \mathrm{CR}$ is exponentially $\mathrm{CR}$-generic if and only if

$$
\lim _{n \rightarrow \infty} \frac{\gamma(n, Q)}{\gamma(n, \mathrm{CR})}=1
$$

with exponentially fast convergence.

Definition 4.3. An automorphism $\tau: F \rightarrow F$ is called a relabeling automorphism if the restriction $\left.\tau\right|_{A_{2 k}}$ is a permutation of $A_{2 k}$. 
Convention 4.4. For the remainder of the paper we adopt the following convention. If $r \geq 0$ is a real number, by saying that $w$ is a word of length $r$ we will mean that $w$ is a word of length $\lfloor r\rfloor$.

Lemma 4.5. Let $0<\lambda<1 / 3$. Let $\tau$ be a nontrivial relabeling automorphism of $F$.

Define $S(\lambda, \tau)$ as the set of all cyclically reduced words $x$ such that $x$ and some cyclic permutation of $\tau(x)$ have a common initial segment of length $\geq \lambda|x|$.

Then $S(\lambda, \tau)$ is exponentially CR-negligible.

Proof. Suppose $x \in S(\lambda, \tau)$ and $|x|=n>1$. Then there exist an initial segment $u$ of $x$ with $|u|=\lambda n$ and a cyclic permutation $v$ taking $\tau(x)$ to $x^{\prime}$ such that $u$ is also an initial segment of $x^{\prime}$.

Case 1. Suppose first that $v$ is a trivial cyclic permutation. Then $u$ is an initial segment of $\tau(x)$ and $u=\tau(u)$. Since $\tau$ is a relabeling automorphism, this implies that there is some letter $a \in A_{2 k}$ such that $a^{ \pm 1}$ does not occur in $u$. Then the number of possibilities for $u$ is at most $2 k(2 k-3)^{\lambda n-1}$ and the number of possibilities for $v$ is at most $2 k(2 k-1)^{(1-\lambda) n-1}$. Hence the number of all such $u$ is at most

$$
\frac{4 k^{2}}{(2 k-1)(2 k-3)}(2 k-3)^{\lambda n}(2 k-1)^{(1-\lambda) n},
$$

which is exponentially smaller than $(2 k-1)^{n}$.

Case 2. Suppose now that $v$ is a nontrivial cyclic permutation, so that $v$ has "translation length" $l \neq 0(\bmod n), 1 \leq l \leq n-1$. Thus $\tau(x)=y_{1} y_{2}, x^{\prime}=y_{2} y_{1}$ and $\left|y_{2}\right|=l$ and $\left|y_{1}\right|=n-l$.

The idea is that there are at least $\lambda n / 6$ letters of $x$ for which there is no choice and which are predetermined by the rest of $x$. Hence the number of possibilities for $x$ is exponentially smaller than $(2 k-1)^{n}$. There are basically two cases: when the overlap between the positions of $u$ in $x$ and in $\tau(x)$ is small (that is both $l$ and $-l$ are large $\bmod n$ ) and when the overlap is large (that is one of $l,-l$ is small $\bmod n$ ).

Subcase 2.A. Assume first that $l, n-l \geq|u| / 6=\lambda n / 6$, so that the overlap between the positions of $u$ in $x$ and $\tau(x)$ has length at most $|u| / 6$.

Then $y_{2}=u y_{2}^{\prime}$ and $\tau(x)=y_{1} u y_{2}^{\prime}$ where $\left|y_{1}\right| \geq|u|$. Hence $x=u v=$ $u v_{1} \tau^{-1}(u) v_{2}$ where $\left|u v_{1}\right|=\left|y_{1}\right|$. We see that in this case the segment $u^{\prime}=\tau(u)$ of $x$ of length $\lambda n / 6$ occurring in the same position in $x$ as $u$ does in $\tau(x)$ is uniquely determined (for a fixed $l$ ) by the rest of the word $x$. The number of choices for $l$ is at most $n$. Given $l$ the number of choices for $\left(u v_{1}, v_{2}\right)$ is at most $\frac{(2 k)^{2}}{(2 k-1)^{2}}(2 k-1)^{n-\lambda n / 6}$. Hence the number of possibilities for such $u$ is at most

$$
n \frac{(2 k)^{2}}{(2 k-1)^{2}}(2 k-1)^{n(1-\lambda / 6)}
$$


which is exponentially smaller than $(2 k-1)^{n}$.

Subcase 2.B. Suppose now that $0<l<|u| / 6$ or $0<n-l<|u| / 6$.

We will assume that $0<l<|u|$ as the other case is similar. Thus $x=u v$ and $\tau(x)=y_{1} u y_{2}$ with $\left|y_{1}\right|=l$. So the positions in which $u$ occurs in $x$ and in $\tau(x)$ have an overlap of length $|u|-l$. That is we can write $u=z_{1} u_{1}$ with $\left|u_{1}\right|=l$

Represent $|u|=m_{0} l+d_{0}$ with $0 \leq d_{0}<l$. Note that $m_{0} \geq 5$ and $d_{0}<l \leq$ $|u| / 6=\lambda n / 6$.

Now write $u$ as

$$
u=z^{\prime} u_{m_{0}} u_{m_{0}-1} \ldots u_{1}
$$

where $\left|u_{i}\right|=l$ for $i=1, \ldots, m_{0}$ and $\left|z^{\prime}\right|=d_{0}$. Since

$$
x=u v=z^{\prime} u_{m_{0}} u_{m_{0}-1} \ldots u_{2} u_{1} v
$$

and

$$
\tau(x)=y_{1} u y_{2}=y_{1} z^{\prime} u_{m_{0}} u_{m_{0}-1} \ldots u_{2} u_{1} y_{2},
$$

and $\left|y_{1}\right|=l$, we see that

$$
u_{2}=\tau\left(u_{1}\right), u_{3}=\tau\left(u_{2}\right)=\tau^{2}\left(u_{1}\right), \ldots u_{m_{0}}=\tau\left(u_{m_{0}-1}\right)=\tau^{m_{0}-1}\left(u_{1}\right) .
$$

Thus, given $l$, the words $u_{1}$ and $z^{\prime}$ determine uniquely the rest of the word $u$, namely the word $w=u_{m_{0}} \ldots u_{2}$. Recall that $\left|z^{\prime}\right| \leq l,\left|u_{1}\right|=l$ and hence

$$
|w| \geq|u|-2 l \geq|u|-2|u| / 6=2|u| / 3=2 \lambda n / 3 .
$$

Recall that $\left|z^{\prime}\right|=d_{0}$ is determined by $l$. So, given $l$ (for which there are at most $n$ choices), the word $w$ is uniquely determined by the rest of the word $x$.

Hence the number of possibilities for $x$ is at most

$$
n \frac{(2 k)^{2}}{(2 k-1)^{2}}(2 k-1)^{n-2 \lambda n / 3}
$$

which is exponentially smaller than $(2 k-1)^{n}$.

By summing up the numbers of possibilities for $x$ in the above cases we see that

$$
\lim _{n \rightarrow \infty} \frac{\gamma(n, S(\lambda, \tau))}{(2 k-1)^{n}}=0
$$

with exponentially fast convergence.

Hence $S(\lambda, \tau)$ is exponentially CR-generic by Proposition 4.2.

The same type of an argument as in the proof of Lemma 4.5 yields the following result. 
Lemma 4.6. Let $0<\lambda<1 / 3$. Let $\tau$ be a nontrivial relabeling automorphism of $F$.

Define $S^{\prime}(\lambda, \tau)$ as the set of all cyclically reduced words $x$ such that $x$ and some cyclic permutation of $\tau\left(x^{-1}\right)$ have a common initial segment of length $\geq \lambda|x|$. Then $S^{\prime}(\lambda, \tau)$ is exponentially CR-negligible.

Definition 4.7. Let $0<\lambda<1 / 3$. For a non-proper power cyclically reduced word $x$ let $y(x, \lambda)$ be the set of all $y$ satisfying one of the following:

(1) the word $y$ is a cyclic permutation of $\tau(x)$ for some nontrivial relabeling automorphism $\tau$;

(2) the word $y$ is a cyclic permutation of $\tau\left(x^{-1}\right)$ for some (possibly trivial) trivial relabeling automorphism $\tau$;

(3) the word $y$ is obtained by a nontrivial cyclic permutation of $x$.

Lemma 4.8. Let $0<\lambda<1 / 3$. Define $E(\lambda)$ as the set of all non-proper power cyclically reduced words $x$ such that for every $y \in \mathcal{y}(x, \lambda)$ the lengths of the maximal common initial segment of $x$ and $y$ is $<\lambda|x|$. Then $E(\lambda)$ is exponentially $\mathrm{CR}$-generic.

Proof. As proved by Arzhantseva and Ol'shanskii [1] (and easy to see directly by arguments similar to those used in Lemma 4.5 and Lemma 4.6), the set of non-proper power cyclically reduced words $x$ whose symmetrized closures satisfy the $C^{\prime}(\lambda)$ small cancellation condition (see [32] for definitions) is exponentially CR-generic. Since there are only finitely many relabeling automorphisms, the result now follows from Lemma 4.5 and Lemma 4.6 by intersecting a finite number of exponentially CR-generic sets.

Remark 4.9. Note that by definition the set $E(\lambda)$ is closed under taking inverses, cyclic permutations and applying relabeling automorphisms. Let $M$ be the number of all (including the trivial one) relabeling automorphisms. Then for any $x \in E(\lambda)$ the set $y(x, \lambda)$ contains exactly $2 M|x|-1$ distinct elements.

\section{Delzant's $T$-invariant for one-relator groups}

Definition 5.1 (Non-reduced $T$-invariant). For a finite group presentation $\Pi=$ $\langle X \mid R\rangle$ denote $\ell_{1}(\Pi):=\sum_{r \in R}|r|$.

If $G$ is a finitely presentable group, define

$$
T_{1}(G):=\min \left\{\ell_{1}(\Pi): \Pi \text { is a finite presentation of } G\right\} .
$$

We call $T_{1}(G)$ the non-reduced $T$-invariant of $G$. 
Obviously, for any $\Pi$ we have $\ell(\Pi) \leq \ell_{1}(\Pi)$ and hence for every finitely presentable group $G$ we have $T(G) \leq T_{1}(G)$. It turns out that under some mild assumptions there is a similar inequality in the other direction:

Lemma 5.2. Let $G$ be a finitely presentable group with no elements of order two. Then there exists a finite presentation $\Pi$ of $G$ such that $\ell(\Pi)=T(G)$ and such that every relation in $\Pi$ has length at least three, and therefore $T_{1}(G) \leq \ell_{1}(\Pi) \leq$ $3 \ell(\Pi)=3 T(G)$.

Consequently,

$$
T(G) \leq T_{1}(G) \leq 3 T(G)
$$

Proof. Among all finite presentations $\Pi$ of $G$ with $\ell(\Pi)=T(G)$, choose a presentation $\Pi=\langle X \mid R\rangle$ of minimal $\ell_{1}$-length.

We claim that every relation in $\Pi$ has length at least three. Clearly, the minimality assumptions on $\Pi$ imply that $\Pi$ has no relations of length one. Suppose $\Pi$ has a relation $r$ of length two. Thus $r=x y$ where $x, y \in X^{ \pm 1}$. We may assume that $y \in X$.

If $x \neq y$ in $F(X)$, let $\Pi^{\prime}$ be the presentation obtained from $\Pi$ by the Tietze transformation consisting of replacing every occurrence of $y$ in the relators of $R$ different from $r$ by $x^{-1}$, freely reducing the resulting relators if needed, then removing the relator $x y$ and removing the generator $y$ from $X$. Then $\ell\left(\Pi^{\prime}\right) \leq \ell(\Pi)=T(G)$ and hence $\ell\left(\Pi^{\prime}\right)=T(G)$. By construction, $\ell_{1}\left(\Pi^{\prime}\right)<\ell_{1}(\Pi)$ contradicting the minimality of $\Pi$.

If $r=x^{2}$, the assumption that $G$ has no elements of order two implies that $x=1$ in $G$. Let $\Pi^{\prime \prime}$ be the presentation obtained from $\Pi$ by removing the generator $x$ from $X$, removing the relation $r=x^{2}$ and deleting all the occurrences of $x$ from the other relations of $R$ and freely reducing the results if necessary. We again have $\ell\left(\Pi^{\prime \prime}\right)=\ell(\Pi)=T(G)$ and hence $\ell\left(\Pi^{\prime \prime}\right)=T(G)$. By construction $\ell_{1}\left(\Pi^{\prime \prime}\right)<$ $\ell_{1}(\Pi)$, contradicting the choice of $\Pi$.

Thus every relation in $\Pi$ has length at least three, as claimed.

Recall that, as specified in Convention 3.1, $k>1$ is a fixed integer and $F=$ $F\left(a_{1}, \ldots, a_{k}\right)$. As before we identify $F$ with the set of all freely reduced words in the alphabet $A_{2 k}=\left\{a_{1}, \ldots, a_{k}, a_{1}^{-1}, \ldots, a_{k}^{-1}\right\}$. For $u \in F$ we denote by $G_{u}$ the one-relator group $G_{u}:=\left\langle a_{1}, \ldots, a_{k} \mid u=1\right\rangle$. If $\Pi$ is a presentation, $G(\Pi)$ denotes the group presented by $\Pi$.

We now recall an important result about isomorphism rigidity of generic onerelator groups that we obtained in [30].

Theorem 5.3 ([30]). Let $k>1$ be a fixed integer and $F=F\left(a_{1}, \ldots, a_{k}\right)$. There exists an exponentially $\mathrm{CR}$-generic set $Q_{k} \subseteq \mathrm{CR}$ with the following properties: 
(1) There is an exponential time algorithm which, given $w \in F$, decides whether or not $w \in Q_{k}$.

(2) The set $Q_{k}$ is closed under taking cyclic permutations, inverses and applying relabeling automorphisms.

(3) Each $u \in Q_{k}$ is minimal in its $\operatorname{Aut}(F)$-orbit, that is $|u| \leq|\alpha(u)|$ for any $\alpha \in \operatorname{Aut}(F)$.

(4) If $r \in Q_{k}$ then $G_{r}$ is torsion-free freely indecomposable non-elementary wordhyperbolic group.

(5) If $u \in Q_{k}$ and $v \in F$ are such that $|u|=|v|$ and $\operatorname{Aut}(F) u=\operatorname{Aut}(F) v$ then $v \in Q_{k}$ and there is a relabeling automorphism $\tau$ of $F$ such that $v$ is a cyclic permutation of $\tau(u)$.

(6) Let $u \in Q_{k}$ and $v \in F$ be such that $|u|=|v|$. Then $G_{u} \cong G_{v}$ if and only if $v \in \mathrm{CR}$ and there is a relabeling automorphism $\tau$ of $F$ such that $v$ is a cyclic permutation of $\tau(u)$ or $\tau(u)^{-1}$.

(7) If $u \in Q_{k}$ and $v \in F$ then $G_{u} \cong G_{v}$ if and only if there is $\alpha \in \operatorname{Aut}(F)$ such that $\alpha(v)=u$ or $\alpha(v)=u^{-1}$.

The following lemma is just the "general enumeration argument".

Lemma 5.4. Let $\mathcal{C}$ be a recursively enumerable class of finite presentations of groups. There is a partial algorithm $\Omega(\mathcal{C})$ which, when given a finite presentation $\Pi=$ $\langle X \mid R\rangle$, finds a finite presentation $\Pi^{\prime} \in \mathcal{C}$ such that $G\left(\Pi^{\prime}\right)$ is isomorphic to $G(\Pi)$ if such a presentation $\Pi^{\prime}$ exists.

Proof. We assume that the generating sets for $\Pi$ and for all presentations in $\mathcal{C}$ are initial segments of a fixed recursive set $\left\{x_{1}, x_{2}, \ldots\right\}$ of generators. We enumerate all tuples $\left(\Pi^{\prime}, b, h, h^{\prime}\right)$ where

$$
\Pi^{\prime}=\left\langle X^{\prime} \mid R^{\prime}\right\rangle \in \mathcal{C}, \quad d \in \mathbb{N}^{+}, \quad h: X \longrightarrow F\left(X^{\prime}\right), \quad h^{\prime}: X^{\prime} \longrightarrow F(X) .
$$

When such a tuple is enumerated, we then enumerate the first $d$ elements of $N^{\prime}=\operatorname{ncl}\left(R^{\prime}\right) \subset F\left(X^{\prime}\right)$ and of $N=\operatorname{ncl}(R) \subset F(X)$. We then check if all of the following hold using only the elements of $N$ and $N^{\prime}$ which have just been enumerated:

$$
\begin{array}{rlrl}
h(r) & \in N^{\prime} & & \text { for all } r \in R, \\
h^{\prime}(r) \in N & & \text { for all } r \in R^{\prime}, \\
h^{\prime} h(x) x^{-1} \in N & & \text { for all } x \in X, \\
h h^{\prime}(x) x^{-1} & \in N^{\prime} & & \text { for all } x \in X^{\prime} .
\end{array}
$$

If all of these memberships are witnessed by the elements of $N$ and $N^{\prime}$ just enumerated, then $h$ and $h^{\prime}$ define mutually inverse isomorphisms between $G(\Pi)$ and $G\left(\Pi^{\prime}\right)$ and we output $\Pi^{\prime}$. If not, we go on to the next tuple. 
Convention 5.5. For $0<\lambda<1 / 3$ denote $Q_{k}(\lambda)=Q_{k} \cap E(\lambda)$ where $E(\lambda)$ is as in Lemma 4.8 and $Q_{k}$ is from Theorem 5.3. By Lemma 4.8 and Theorem 5.3 the set $Q_{k}(\lambda)$ is exponentially CR-generic.

Lemma 5.6. There exists a constant $N=N(k)>0$ with the following property. Let $0<\lambda<1 / 3$ be a rational number and let $r \in Q_{k}(\lambda)$ be a nontrivial cyclically reduced word and $G_{r}:=\left\langle a_{1}, \ldots, a_{k} \mid r=1\right\rangle$. Thus $r$ is not a proper power and it satisfies the $C^{\prime}(\lambda)$ small cancellation condition.

Suppose $G_{r}$ can be presented by a finite presentation

$$
\Pi=\left\langle b_{1}, \ldots b_{m} \mid r_{1}, \ldots, r_{t}\right\rangle
$$

where $t \geq 1$.

$$
\text { Then } C_{2 k}(r) \leq N \ell_{1}(\Pi) \log _{2} \ell_{1}(\Pi)+|r| N \lambda+N \text {. }
$$

Proof. We describe an algorithm $\mathcal{A}$, which, given a presentation ( $\dagger$ ) for $G_{r}$ and an initial segment $u$ of $r$ of length $\lambda|r|$, will recover the word $r$.

First, note that we are assuming that $(\dagger)$ defines a group isomorphic to the $k$ generator one-relator group $G_{r}$ with defining relator in $Q_{k}$. We first apply the algorithm $\Omega(\mathcal{C})$ from Lemma 5.4 with $\mathcal{C}$ the class of all $k$-generator one-relator presentations with defining relators from $Q_{k}$. (Note that $\mathcal{C}$ is recursive by part (1) of Theorem 5.3.) This procedure finds some cyclically reduced word $v \in Q_{k}$ such that $(\dagger)$ defines a group isomorphic to $G_{v}$.

Thus $G_{r} \cong G_{v}$ and both $r$ and $v$ (as well as $v^{-1}$ ) are minimal cyclically reduced words from $Q_{k}$. By Theorem $5.3|v|=|r|$ and there is a relabeling automorphism $\tau$ of $F$ such that $r$ is a cyclic permutation of $\tau(v)$ or $\tau(v)^{-1}$.

Construct the set $B$ consisting of all words $x$ with the property that there is a relabeling automorphism $\tau$ of $F$ such that $x$ is a cyclic permutation of $\tau(v)$ or $\tau(v)^{-1}$. Thus $r \in B$. By Lemma 4.8 there is a unique element of $B$ having the same initial segment of length $\lambda|r|$ as does $r$, namely $r$ itself. Recall that the initial segment $u$ of $r$ of length $\lambda|r|$ is part of the input for algorithm $\mathcal{A}$. Then we list all elements of $B$ and check which one of them has initial segment $u$. That element is $r$.

The algorithm $\Omega(\mathcal{C})$ is fixed. The further input of $\mathcal{A}$, required to compute $r$, consists of the presentation ( $\dagger$ ) and the initial segment $u$ of $r$ with $|u|=\lambda|r|$. We need to estimate the length of this input when expressed as a binary sequence. Put $T=\ell_{1}(\Pi)$. First note that in $(\dagger)$ every $b_{i}$ must occur in some $r_{j}^{ \pm 1}$ since $G_{r}$ is a one-ended group by Theorem 5.3 and therefore $m \leq T$.

We can now encode the presentation ( $\dagger$ ) by writing each subscript $i=1, \ldots, m$ for each occurrence of $b_{i}$ in $(\dagger)$ as a binary integer. Using $\bar{i}$ to denote the binary expression for $i$, we replace each occurrence of $b_{i}$ in (†) by $b \bar{i}$ and each occurrence of $b_{i}^{-1}$ by $-b \bar{i}$. Note that the bit-length of the binary expression $\bar{i}$ of $i$ is at most 
$\log _{2} i$. This produces an unambiguous encoding of $(\dagger)$ as a string $W$ of length at most $O\left(T \log _{2} T\right)$ over the six letter alphabet

$$
\begin{array}{llllll}
b & 0 & 1 & - & -
\end{array}
$$

and this alphabet can then be block-coded into binary in the standard way.

Since the number $k$ of generators is fixed, describing $u$ requires at most $O(|u|)$ number of bits.

Hence there exist a constant $N=N(k)>0$ such that

$$
C_{2 k}(r) \leq N T \log _{2} T+|r| N \lambda+N .
$$

Theorem 5.7. Let $k>1$ be a fixed integer and $F=F\left(a_{1}, \ldots, a_{k}\right)$. For any $\varepsilon$, $0<\varepsilon<1$ there is an integer $n_{1}>0$ and a constant $M=M(k, \varepsilon)>0$ with the following property.

Let $J$ be the set of all nontrivial cyclically reduced words $r$ such that

$$
T\left(G_{r}\right) \log _{2} T\left(G_{r}\right) \geq M|r| .
$$

Then for any $n \geq n_{1}$

$$
\frac{\gamma(n, J)}{\gamma(n, \mathrm{CR})} \geq 1-\varepsilon
$$

Proof. Let $N>0$ be the constant provided by Lemma 5.6. Choose a rational number $\lambda, 0<\lambda<1 / 3$, so that $\frac{\log _{2}(2 k-1)}{2}-N \lambda>0$.

Let $c>0$ be an arbitrary integer. Let $n_{0}>1$ be the integer provided by Proposition 3.3. As in Proposition 3.3 let $Z$ be the set of all cyclically reduced words $x$ of length $\geq n_{0}$ such that

$$
C_{2 k}(x) \geq-\frac{c}{2}+|x| \frac{\log _{2}(2 k-1)}{2} .
$$

Then by Proposition 3.3 for any $n \geq n_{0}$ we have

$$
\frac{\gamma(n, Z)}{\gamma(n, \mathrm{CR})} \geq 1-\frac{1}{2^{c}}
$$

Since $Q_{k}(\lambda)$ is exponentially generic, Proposition 4.2 implies that there is $n_{1} \geq n_{0}$ such that for any $n \geq n_{1}$

$$
\frac{\gamma\left(n, Z \cap Q_{k}(\lambda)\right)}{\gamma(n, \mathrm{CR})} \geq 1-2 \frac{1}{2^{c}} .
$$

Now suppose $r \in Z \cap Q_{k}(\lambda)$ and $|r| \geq n_{1}$. 
Then by Lemma 5.6

$$
-\frac{c}{2}+|r| \frac{\log _{2}(2 k-1)}{2} \leq C_{2 k}(r) \leq N T_{1}\left(G_{r}\right) \log _{2} T_{1}\left(G_{r}\right)+|r| N \lambda+N,
$$

and hence by Lemma 5.2

$$
\begin{aligned}
|r|\left(\frac{\log _{2}(2 k-1)}{2}-N \lambda\right)-N-\frac{c}{2} & \leq N T_{1}\left(G_{r}\right) \log _{2} T_{1}\left(G_{r}\right) \\
& \leq 3 N T\left(G_{r}\right) \log _{2} 3 T\left(G_{r}\right) \\
& =3 N T\left(G_{r}\right)\left(\log _{2} T\left(G_{r}\right)+\log _{2} 3\right) \\
& \leq 30 N T\left(G_{r}\right) \log _{2} T\left(G_{r}\right),
\end{aligned}
$$

yielding the conclusion of the theorem.

We need the following result of Rivin on the precise number of cyclically reduced words of a given length.

Proposition 5.8. For any $n \geq 1$ we have

$$
\gamma(n, \mathrm{CR})=(2 k-1)^{n}+1+(k-1)\left[1+(-1)^{n}\right] .
$$

Thus for a fixed $k \geq 2$ we have $\gamma(n, \mathrm{CR}) \sim(2 k-1)^{n}$.

The next statement is obvious.

Lemma 5.9. The number of relabeling automorphisms is $k ! 2^{k}$.

Theorem 5.10. Fix an integer $k \geq 2$. Let $I_{n}$ be the number of isomorphism types of groups admitting a $k$-generator one-relator presentation where the defining relator is cyclically reduced and has length $n$. Then

$$
I_{n} \sim \frac{(2 k-1)^{n}}{n k ! 2^{k+1}} .
$$

Proof. Choose $0<\lambda<1 / 3$ so that $Q_{k}(\lambda) \subseteq$ CR is exponentially CR-generic. Recall that $Q_{k}(\lambda)$ is closed under applying inverses, cyclic permutations and relabeling automorphisms.

Denote by $M=k ! 2^{k}$ the number of all relabeling automorphisms of $F=$ $F\left(a_{1}, \ldots, a_{k}\right)$.

By Remark 4.9 for any $u \in Q_{k}(\lambda)$ we have $\# y(u, \lambda)=2 M|u|-1$. Hence by Theorem 5.3 the number of all $v \in Q_{k}(\lambda)$ with $G_{u} \cong G_{v}$ is equal to $2 M|u|$. Therefore the set of words of length $n$ in $Q_{k}(\lambda)$ defines precisely $\frac{\gamma\left(n, Q_{k}(\lambda)\right)}{2 M n}$ isomorphism types of one-relator groups. Denote $b_{n}=\gamma(n, \mathrm{CR})-\gamma\left(n, Q_{k}(\lambda)\right)$. Thus $\frac{b_{n}}{(2 k-1)^{n}} \rightarrow 0$ exponentially fast as $n \rightarrow \infty$. 
Hence

$$
\left|I_{n}-\frac{\gamma\left(n, Q_{k}(\lambda)\right)}{2 M n}\right| \leq b_{n}
$$

and so

$$
\left|\frac{2 n M I_{n}}{(2 k-1)^{n}}-\frac{\gamma\left(n, Q_{k}(\lambda)\right)}{(2 k-1)^{n}}\right| \leq \frac{2 M n b_{n}}{(2 k-1)^{n}} .
$$

By CR-genericity of $Q_{k}(\lambda)$ and by Rivin's formula we have

$$
\lim _{n \rightarrow \infty} \frac{\gamma\left(n, Q_{k}(\lambda)\right)}{(2 k-1)^{n}}=\lim _{n \rightarrow \infty} \frac{\gamma\left(n, Q_{k}(\lambda)\right)}{\gamma(n, \mathrm{CR})} \frac{\gamma(n, \mathrm{CR})}{(2 k-1)^{n}}=1 \cdot 1=1 .
$$

Since $\lim _{n \rightarrow \infty} \frac{2 M n b_{n}}{(2 k-1)^{n}}=0$, this implies

$$
\lim _{n \rightarrow \infty} \frac{2 n M I_{n}}{(2 k-1)^{n}}=1,
$$

and hence

$$
I_{n} \sim \frac{(2 k-1)^{n}}{2 M n}=\frac{(2 k-1)^{n}}{n k ! 2^{k+1}}
$$

as required.

\section{References}

[1] G. Arzhantseva and A. Ol'shanskii, Generality of the class of groups in which subgroups with a lesser number of generators are free. Mat. Zametki 59 (1996), 489-496; English transl. Math. Notes 59 (1996), 350-355. Zbl 0877.20021 MR 1445193

[2] G. Arzhantseva, On groups in which subgroups with a fixed number of generators are free. Fundam. Prikl. Mat. 3 (1997), 675-683 (in Russian). Zbl 0929.20025 MR 1794135

[3] G. Arzhantseva, Generic properties of finitely presented groups and Howson's theorem. Comm. Algebra 26 (1998), 3783-3792. Zbl 0911.20027 MR 1647075

[4] G. Arzhantseva, A property of subgroups of infinite index in a free group. Proc. Amer. Math. Soc. 128 (2000), 3205-3210. Zbl 0976.20014 MR 1694447

[5] M. Bestvina and M. Feighn, Bounding the complexity of simplicial group actions on trees. Invent. Math. 103 (1991), 449-469 Zbl 0724.20019 MR 1091614

[6] M. Bestvina and M. Feighn, A counterexample to generalized accessibility. In Arboreal group theory (Berkeley, CA, 1988), Math. Sci. Res. Inst. Publ. 19, Springer-Verlag, New York 1991, 133-141. Zbl 0826.20027 MR 1105331

[7] B. H. Bowditch, Cut points and canonical splittings of hyperbolic groups. Acta Math. 180 (1998), no. 2, 145-186. Zbl 0911.57001 MR 1638764

[8] B. H. Bowditch, Boundaries of strongly accessible hyperbolic groups. In The Epstein birthday schrift (I. Rivin et al., eds.), Geom. Topol. Monogr. 1, Geometry \& Topology Publications, Coventry 1998, 51-97 (electronic). Zbl 0918.20027 MR 1668331 
Vol. 80 (2005)

[9] C. Champetier, Petite simplification dans les groupes hyperboliques. Ann. Fac. Sci. Toulouse Math. (6) 3 (1994), 161-221. Zbl 0803.53026 MR 1283206

[10] C. Champetier, Propriétés statistiques des groupes de présentation finie. Adv. Math. 116 (1995), 197-262. Zbl 0847.20030 MR 1363765

[11] C. Champetier, The space of finitely generated groups. Topology 39 (2000), 657-680. Zbl 0959.20041 MR 1760424

[12] P.-A. Cherix and A. Valette, On spectra of simple random walks on one-relator groups. With an appendix by Paul Jolissaint. Pacific J. Math. 175 (1996), 417-438. Zbl 0865.60059 MR 1432838

[13] P.-A. Cherix and G. Schaeffer, An asymptotic Freiheitssatz for finitely generated groups. Enseign. Math. (2) 44 (1998), 9-22. Zbl 0987.20012 MR 1643258

[14] T. Delzant, Décomposition d'un groupe en produit libre ou somme amalgamée, J. Reine Angew. Math. 470 (1996) 153-180. Zbl 0836.20038 MR 1370211

[15] T. Delzant, Sur l'accessibilité acylindrique des groupes de présentation finie. Ann. Inst. Fourier (Grenoble) 49 (1999), , 1215-1224. Zbl 0999.20017 MR 1703085

[16] T. Delzant and L. Potyagailo, Accessibilité hiérarchique des groupes de présentation finie. Topology 40 (2001), 617-629. Zbl 0996.20027 MR 1838998

[17] M. Dunwoody, The accessibility of finitely presented groups. Invent. Math. 81 (1985), 449-457. Zbl 0572.20025 MR 0807066

[18] M. Dunwoody, An inaccessible group. In Geometric group theory (Sussex, 1991), Vol. 1, London Math. Soc. Lecture Note Ser. 181, Cambridge University Press, Cambridge 1993, 75-78. Zbl 0833.20035 MR 1238516

[19] M. J. Dunwoody and M. E. Sageev, JSJ-splittings for finitely presented groups over slender groups. Invent. Math. 135 (1999), 25-44. Zbl 0939.20047 MR 1664694

[20] L. Fortnow, Kolmogorov complexity. In Aspects of complexity (Kaikoura, 2000), de Gruyter Ser. Log. Appl. 4, de Gruyter, Berlin 2001, 73-86. Zbl 1027.68610 MR 1884262

[21] K. Fujiwara and P. Papasoglu, JSJ decompositions and complexes of groups. Preprint, 1996.

[22] R. I. Grigorchuk, A relationship between algorithmic problems and entropy characteristics of groups. Dokl. Akad. Nauk SSSR 284 (1985), 24-29; English transl. Sov. Math. Dokl. 32 (1985), 355-360. Zbl 0596.20022 MR 0806660

[23] M. Gromov, Hyperbolic Groups. In Essays in Group Theory (G. M. Gersten, ed.), Math. Sci. Res. Inst. Publ. 8, Springer-Verlag, New York 1987, 75-263. Zbl 0634.20015 MR 0919829

[24] M. Gromov, Asymptotic invariants of infinite groups. Geometric group theory (Sussex, 1991), Vol. 2, London Math. Soc. Lecture Note Ser. 182, Cambridge University Press, Cambridge 1993. Zbl 0841.20039 MR 1253544

[25] M. Gromov, Random walk in random groups. Geom. Funct. Anal. 13 (2003), 73-146. Zbl 01971826 MR 1978492

[26] I. Grushko, Über die Basen eines freien Produktes von Gruppen. Rec. Math. [Mat. Sb.] N.S. 8 (1940), 169-182. Zbl 0023.30102 MR 0003412

[27] I. Kapovich, A. Myasnikov, P. Schupp and V. Shpilrain, Generic-case complexity, decision problems in group theory and random walks. J. Algebra 264 (2003), 665-694. Zbl 1041.20021 MR 1981427 
[28] I. Kapovich, A. Myasnikov, P. Schupp and V. Shpilrain, Average-case complexity for the word and membership problems in group theory. Adv. Math. 190 (2005), 343-359. Zbl 02126454 MR 2102661

[29] I. Kapovich and P. Schupp, Genericity, the Arzhantseva-Ol'shanskii method and the isomorphism problem for one-relator groups. Math. Ann. 331 (2005), 1-19. Zbl 02132961 MR 2107437

[30] I. Kapovich, P. Schupp and V. Shpilrain, Generic properties of Whitehead's algorithm and isomorphism rigidity of random one-relator groups. Pacific J. Math., to appear; http://front.math.ucdavis.edu/math.GR/0303386.

[31] I. Kapovich and R. Weidmann, Acylindrical accessibility for groups acting on $\mathbb{R}$-trees. Math. Z. 249 (2005), 1-19. Zbl 02156076 MR 2126215

[32] R. Lyndon and P. Schupp, Combinatorial Group Theory. Reprint of the 1977 edition, Classics in mathematics, Springer-Verlag, Berlin 2001. Zbl 0997.20037 MR 1812024

[33] L. Levin, Laws on the conservation (zero increase) of information, and questions on the foundations of probability theory. Problemy Peredaci Informacii 10 (3) (1974), 30-35 (in Russian). Zbl 0312.94007 MR 0469513

[34] M. Li and P. Vitanyi, An Introduction to Kolmogorov Complexity and Its Applications (2nd edition). Grad. Texts in Comput. Sci., Springer-Verlag, New York 1997. Zbl 0866.68051 MR 1438307

[35] Y. Ollivier, Critical densities for random quotients of hyperbolic groups. C. R. Math. Acad. Sci. Paris 336 (2003), 391-394. Zbl 1050.20048 MR 1979351

[36] A. Yu. Ol'shanskii, Almost every group is hyperbolic. Internat. J. Algebra Comput. 2 (1992), 1-17. Zbl 0779.20016 MR 1167524

[37] E. Pervova and C. Petronio, Complexity and T-invariant of Abelian and Milnor groups, and complexity of 3-manifolds. Preprint, 2004; http://front.math.ucdavis. edu/math.GT/0412187

[38] E. Rips and Z. Sela, Cyclic splittings of finitely presented groups and the canonical JSJ decomposition. Ann. of Math. (2) 146 (1997), 53-109. Zbl 0910.57002 MR 1469317

[39] S. Ross, Stochastic Processes (2-nd editon), Wiley Series in Probability and Statistics: Probability and Statistics. John Wiley \& Sons, Inc., New York 1996. Zbl 0888.60002 MR 1373653

[40] Z. Sela, Acylindrical Accessibility. Invent. Math. 129 (1997), 527-565. Zbl 0887.20017 MR 1465334

[41] Z. Sela, Structure and rigidity in (Gromov) hyperbolic groups and discrete groups in rank 1 Lie groups. II. Geom. Funct. Anal. 7 (1997), 561-593. Zbl 0884.20025 MR 1466338

[42] R. Weidmann The Nielsen method for groups acting on trees. Proc. London Math. Soc. (3) 85 (2002), 93-118. Zbl 1018.20020 MR 1901370

[43] A. Zuk, On property (T) for discrete groups. In Rigidity in dynamics and geometry (Cambridge, 2000), Springer-Verlag, Berlin 2002, 473-482. Zbl 1007.22011 MR 1919418

Received May 27, 2003; revised January 30, 2005 
Vol. $80(2005)$

Ilya Kapovich, Department of Mathematics, University of Illinois at Urbana-Champaign, 1409 West Green Street, Urbana, IL 61801, U.S.A.

E-mail: kapovich@math.uiuc.edu

URL: http://www.math.uiuc.edu/ /kapovich/

Paul Schupp, Department of Mathematics, University of Illinois at Urbana-Champaign, 1409

West Green Street, Urbana, IL 61801, U.S.A.

E-mail: schupp@math.uiuc.edu

URL: http://www.math.uiuc.edu/People/schupp.html 